\title{
Optimization of virus-induced gene silencing in pepper (Capsicum annuum L.)
}

\author{
J.-E. Wang ${ }^{1,2}$, D.-W. Li ${ }^{1,2}$, Z.-H. Gong ${ }^{1,2}$ and Y.-L. Zhang ${ }^{1,2}$ \\ ${ }^{1}$ College of Horticulture, Northwest A\&F University, Yangling, Shaanxi, China \\ ${ }^{2}$ State Key Laboratory of Crop Stress Biology for Arid Regions, \\ Northwest A\&F University, Yangling, Shaanxi, China \\ Corresponding author: Z.-H. Gong \\ E-mail: zhgong@nwsuaf.edu.cn
}

Genet. Mol. Res. 12 (3): 2492-2506 (2013)

Received August 28, 2012

Accepted January 26, 2013

Published July 24, 2013

DOI http://dx.doi.org/10.4238/2013.July.24.4

\begin{abstract}
Virus-induced gene silencing is currently a powerful tool for the study of gene function in plants. Here, we optimized the protocol for virus-induced gene silencing, and investigated factors that affect the efficiency of tobacco rattle virus-induced gene silencing in pepper plants. Consequently, an optimal protocol was obtained by the syringe-infiltration method in the leaves of pepper plants. The protocol involves 2-leaf stage plants, preparing the Agrobacterium inoculum at a final $\mathrm{OD}_{600}$ of 1.0 and then growing the inoculated plants at $22^{\circ} \mathrm{C}$. Using this protocol, we achieved high efficiency in silencing $C a P D S$ in different cultivars of pepper plants. We further used the $C a P O D$ gene to illustrate the general reliability of this optimized protocol. Viral symptoms were observed on the leaves of inoculated plants of the Early Calwonder cultivar 25 days post-inoculation, indicating that this protocol can also be used to silence other genes in pepper plants. Real-time polymerase chain reaction analyses revealed that the expression levels of $C a P D S$ and $C a P O D$ were dramatically reduced in inoculated leaves compared to
\end{abstract}


control plants. These results demonstrate that the optimized protocol can be applied to functional genomic studies in pepper to investigate genes involved in a wide range of biological processes.

Key words: Pepper; Optimized protocol; Virus-induced gene silencing; Tobacco rattle virus; Phytoene desaturase

\section{INTRODUCTION}

Pepper (Capsicum annuum L.) is a nutritionally and economically important crop that is cultivated throughout the world as a vegetable, condiment, and food additive. Genetic transformation is extremely difficult in pepper plants, and the efficiency of transformation is very low. Although a number of transformation protocols have been published (Manoharan et al., 1998; Mihalka et al., 2000; Kim et al., 2001), investigations into the functions of pepper genes remain problematic due to the lack of stable and effective genetic transformation procedures. Moreover, transformation itself is a complex, labor-intensive procedure, with low efficiency (Baulcombe, 2004; Benedito et al., 2004; Burch-Smith et al., 2004).

These disadvantages can be avoided by adopting large-scale functional genomic approaches to analyze gene function. Virus-induced gene silencing (VIGS) provides an attractive alternative tool to investigate gene function in plants by knocking down target genes of interest without the need for transformation (Baulcombe, 1999; Dinesh-Kumar et al., 2003). This approach is considerably less time-consuming than classical stable transformation. VIGS developed from RNA-mediated post-transcriptional gene silencing (Baulcombe, 1999), which is a defense system against pathogens and is naturally present in plants and other organisms (DineshKumar et al., 2003; Burch-Smith et al., 2004; Robertson, 2004). When a virus infects a plant tissue and spreads systemically, endogenous gene transcripts with sequence identities matching the engineered sequence in the viral vector (VIGS vector) are degraded by post-transcriptional gene silencing (Baulcombe, 1999). Consequently, the plant defends itself against the virus by degrading the accumulating viral genome and, in the process, silences its own gene.

Recently, great efforts have gone into exploiting VIGS for gene function analyses in a variety of plant species. So far, VIGS has been applied to more than 30 plant species (Becker and Lange, 2010), indicating that it has become a powerful tool for functional gene analysis in plants. More than 30 VIGS vectors have been developed so far, including tobacco mosaic virus (Kumagai et al., 1995), potato virus X (Ruiz et al., 1998), tobacco rattle virus (TRV) (Ratcliff et al., 2001), barley stripe mosaic virus (Holzberg et al., 2002), and brome mosaic virus (Ding et al., 2006). These vectors have been widely used to uncover the functions of genes involved in basic cellular functions, metabolic pathways, development biology, plant-microbe interactions, and abiotic stress (Huang et al., 2012). In particular, the TRV-based vector, TRV, has been widely used in gene silencing studies involving Nicotiana benthamiana, tomato, pepper, eggplant, and other Solanaceous species (Kumagai et al., 1995; Brigneti et al., 2004; Fu et al., 2005; Chung et al., 2004; Liu et al., 2002a, 2004, 2012). In the TRV vector, RNA1 provides the replication and movement functions, and TRV-RNA2 from the isolate PPK20 encodes the viral coat protein (Ratcliff et al., 2001; Liu et al., 2002b). One distinct advantage of using TRV for VIGS is the ability of the virus to spread rapidly throughout the entire plant, including the meristem tissue. VIGS has therefore been used to study gene functions in leaves, 
flowers (Liu et al., 2004; Chen et al., 2004), fruits (Fu et al., 2005; Orzaez et al., 2006), and roots (Valentine et al., 2004).

Despite its successful application, the silencing efficiency of VIGS varies among different plant species and even among cultivars (Burch-Smith et al., 2004; Ghazala and Varrelmann, 2007; MacFarlane, 2010). In addition, many environmental factors influence the efficiency of VIGS, such as the Agrobacterium inoculation method, growth stages of the plants, and the growth temperature. The VIGS system, therefore, requires optimization in order to increase the efficiency of gene silencing studies across a range of plants. The phytoene desaturase $(P D S)$ reporter gene has been widely and successfully used in VIGS studies based on its observed silencing phenotype (Ratcliff et al., 2001; Liu et al., 2002a). Chung et al. (2004) were the first to achieve VIGS of pepper using a TRV-based VIGS system, but a comprehensive optimized system for VIGS is still lacking.

In this study, we used a TRV-based VIGS vector to optimize the protocol of gene silencing in the Early Calwonder (EC) pepper cultivar, using the syringe-infiltration method. The results indicated that the growth stage, the concentration of Agrobacterium inoculum, and the growth temperature for inoculated plants were important factors in determining the efficiency of gene silencing. Following this optimized protocol, we achieved efficient gene silencing of the reporter gene, $C a P D S$, in different cultivars of pepper plants. This study should provide a convenient, fast, and effective method for large-scale gene silencing in pepper functional genomics.

\section{MATERIAL AND METHODS}

\section{Plant material and growth conditions}

Pepper plants were used for all experiments. Seeds were grown in a plastic tray containing a steam-sterilized soil mixture. The growth chamber conditions were maintained at $25^{\circ} / 18^{\circ} \mathrm{C}$ under a 16 -h light $/ 8$-h dark photoperiod cycle with $60 \%$ relative humidity.

\section{Plasmid construction}

The pTRV1 and pTRV2 VIGS vectors (Liu et al., 2002a) were kindly provided by Dr. Dinesh-Kumar of Yale University. Two constructs were used in this study, including pTRVCaPDS (CaPDS, phytoene desaturase from C. annuum, GenBank accession No. X68058.1) and pTRV-CaPOD (CaPOD, peroxidase from C. annuum, GenBank accession No. FJ596178).

pTRV-CaPDS was constructed from a 324-bp fragment of CaPDS, which was polymerase chain reaction (PCR)-amplified from a pepper cDNA template using 2 primers (forward: 5'-GGGGAATTCTGTTGTCAAAACTCCAAGGTCTGTA-3' with an EcoRI restriction site and reverse: 5'-GGGGGATCCTTTCTCCCACTTGGTTCACTCTTGT-3' with a $B a m \mathrm{HI}$ restriction site). The resulting product was cloned into the EcoRI-BamHI-cut pTRV2 to form pTRV-CaPDS.

pTRV-CaPOD was constructed from a 485-bp fragment of $C a P O D$, which was PCRamplified from a pepper cDNA template using 2 primers (forward: 5'-GGGTCTAGAGTGCT CAACACACACTTTATTCTTCTC-3' with an XbaI restriction site and reverse: 5'-GGGGGA TCCCCAAGAATGACAACAGAGTCCCTA-3' with a BamHI restriction site). The resulting product was cloned into the $X b a \mathrm{I}-\mathrm{Bam} \mathrm{HI}$-cut pTRV2 to form pTRV-CaPOD. 
Sequences of all pTRV2 derivatives were confirmed by sequencing. For the VIGS assay, either pTRV1 or pTRV2 and its derivatives were introduced into A. tumefaciens strain GV3101 by the freeze-thaw method.

\section{Agrobacterium inoculation}

Agrobacterium strain GV3101 containing pTRV1 or pTRV2 and its derivatives were used for the VIGS experiments. Cultures $(5 \mathrm{~mL})$ were grown in Luria-Bertani medium containing the appropriate antibiotic $(50 \mathrm{mg} / \mathrm{L}$ kanamycin, $50 \mathrm{mg} / \mathrm{L}$ rifampicin, and $50 \mathrm{mg} / \mathrm{L}$ gentamycin), and shaken at $28^{\circ} \mathrm{C}$ overnight. The next day, each Agrobacterium culture was inoculated into $50 \mathrm{~mL}$ Luria-Bertani medium containing the same antibiotics, $10 \mathrm{mM}$ 2-(4-morpholino)ethane sulfonic acid (MES), and $200 \mu \mathrm{M}$ acetosyringone, and grown in a shaker at $200 \mathrm{rpm}$. After 24 h, Agrobacterium cells were harvested and resuspended in the Agrobacterium infiltration buffer ( $10 \mathrm{mM} \mathrm{MgCl}_{2}, 10 \mathrm{mM}$ MES, pH 5.6, $200 \mu \mathrm{M}$ acetosyringone). The Agrobacterium cells were collected by centrifugation ( $3000 \mathrm{rpm}, 10 \mathrm{~min}$ ), suspended in infiltration medium ( $20 \mathrm{mM}$ citric acid, $2 \%$ sucrose, $\mathrm{pH} 5.6$ ) and adjusted to $\mathrm{OD}_{600}$ values between 0.5 and 4.0. The suspensions of Agrobacterium inocula containing pTRV1 and pTRV2 or its derivatives were mixed at a 1:1 ratio and incubated for $3 \mathrm{~h}$ at room temperature. The Agrobacterium was infiltrated into the cotyledons of different growth stages $(2,4$, and 6 true leaves) using a $1-\mathrm{mL}$ sterilized syringe without a needle (Liu et al., 2002a). The Agrobacterium-infiltrated pepper plants were transferred to $18^{\circ} \mathrm{C}$ for 2 days and grown in a growth chamber at different temperatures $\left(22^{\circ}\right.$, $25^{\circ}, 28^{\circ} \mathrm{C}$ ) under a 16 -h light $/ 8$-h dark photoperiod cycle with $60 \%$ relative humidity.

\section{RNA isolation and real-time quantitative PCR (qRT-PCR) analysis}

qRT-PCR was used to quantify the abundances of $C a P D S$ and $C a P O D$ transcripts in the leaves of inoculated pepper plants. Total RNA was extracted from leaves of silenced and non-silenced (infiltrated with empty vectors of pTRV1 and pTRV2) 15 days after infiltration using Trizol reagents (Invitrogen), following manufacturer protocols. The first-strand cDNA was synthesized from $500 \mathrm{ng}$ total RNA using a SYBR ${ }^{\circledR}$ Premix Ex Taq ${ }^{\mathrm{TM}}$ II kit (TaKaRa). Primers that annealed outside the region targeted for silencing were used to ensure that only the endogenous gene was being tested. The ubiquitin gene, ubi3, served as an internal control gene. The primers specific to a 120 -bp fragment of $u b i 3$ were 5'-TGTCCATCTGCTCTCTGTTG$3^{\prime}$ and 5'-CACCCCAAGCACAATAAGAC-3'. Primers used in RT-PCR for CaPDS were 5'-AGGTCTTCTTTGGGAACTGAT-3' and 5'-GTTATGGAATGGGGATTACGA-3'. The primers used for qRT-PCR for CaPOD were 5'-CAAGGTTCAATGTGTGTTACC-3' and 5'-ATGATGATACAAATACAGTGCC-3'. qRT-PCR was performed following manufacturer protocols in the iQ5 Real-Time PCR Detection System and analyzed using the iQ5 Standard Edition Optical System Software Version 2.1 (Bio-Rad Laboratories, Inc., Hercules, CA, USA). Three technical replicates were run for each biological replicate.

\section{Chlorophyll content measurement}

Chlorophyll was measured immediately after preparing the extracts, as described by Lichtenthaler(1987) and Hajirezaei et al. (2002), with slight modifications. Leaves (approximately 
$0.1 \mathrm{~g}$ ) were extracted in $80 \%$ (v/v) aqueous acetone, followed by an additional step with $100 \%$ acetone. Samples were then incubated at room temperature until they were white in appearance. Absorbance was recorded at 663 and $645 \mathrm{~nm}$ using a UV-visible spectrophotometer. Total chlorophyll was expressed as the percentage reduction relative to the corresponding control.

\section{Statistical analysis}

The data were subjected to analysis of variance using the JMP software, version 4.0.4 (SAS Institute Inc., Cary, NC, USA). When a significant F-test was obtained $(\mathrm{P} \leq 0.05)$, separation of treatment means was accomplished by Fisher's protected least significant difference. Values are reported as means $\pm \mathrm{SD}$ of 3 independent experiments.

VIGS efficiency analysis was conducted in triplicate with 30 plants per experiment. The efficiency of gene silencing was calculated with the following formula: gene silencing efficiency $(\%)=$ (number of plants showing the photobleaching phenotype / total number of tested plants) $\mathrm{x} 100 \%$.

\section{RESULTS}

To estimate the efficiency of VIGS in pepper, we used the PDS gene from pepper in a pTRV2 vector. $P D S$ encodes an enzyme involved in the carotenoid biosynthesis pathway (Cunningham and Gantt, 1998). The silencing of PDS has previously been used as a visual marker for the effectiveness of VIGS in several plants, such as tomato (Liu et al., 2002a), Arabidopsis thaliana (Turnage et al., 2002), N. benthamiana (Ratcliff et al., 2001), and $C$. annuum L. (Chung et al., 2004). The silencing of PDS produces a photobleaching phenotype, which occurs in the absence of the $P D S$ gene product (Turnage et al., 2002). In this study, a 324-bp fragment of the $C a P D S$ gene was obtained through RT-PCR, which was used to form a pTRV2-CaPDS construct in order to silence the $P D S$ gene in pepper. The effects of different factors on VIGS efficiency in pepper plants were then estimated.

\section{Effect of different cultivars of pepper on VIGS efficiency in agroinoculated plants}

Cultivars of the same species often vary in their susceptibility to TRV infection (Ghazala and Varrelmann, 2007; MacFarlane, 2010) and in TRV-VIGS efficiency (BurchSmith et al., 2004; Deng et al., 2012; Bennypaul et al., 2012). To estimate the efficiency of VIGS in different cultivars of pepper, we examined gene silencing in 3 agroinoculated cultivars of pepper: CM334 (A5), PBC137 (A3), and EC. The Agrobacterium inoculum was infected into 4-leaf stage plants with a final $\mathrm{OD}_{600}$ of 1.0. Next, Agrobacterium-inoculated plants were transferred to $18^{\circ} \mathrm{C}$ for 2 days and grown in a growth chamber at $22^{\circ} \mathrm{C}$ under a 16-h light/8-h dark photoperiod cycle with $60 \%$ relative humidity. The photobleaching symptom was observed on the leaves of infected pepper plants 15 days postinoculation. The efficiency of VIGS in the different pepper cultivars is shown in Figure 1. EC exhibited the most intensive $P D S$-silencing phenotype and had the highest observed gene-silencing efficiency of $94 \%$, while A5 and A3 showed gene silencing efficiencies of 64 and $77 \%$, respectively. Based on these results $\mathrm{EC}$ was used as the target plant for agroinoculation in all subsequent gene silencing experiments. 


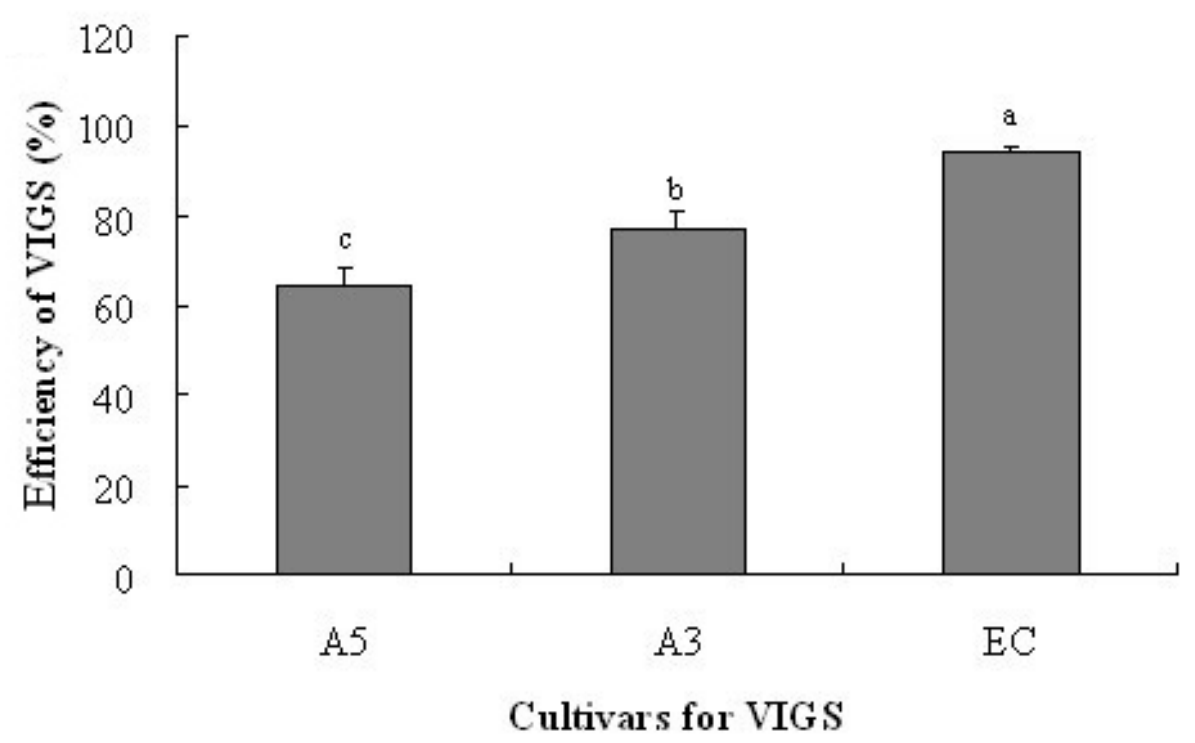

Figure 1. Effect of different cultivars for agroinoculated plants on virus-induced gene silencing (VIGS) efficiency in pepper. The Arobacterium inocula with $\mathrm{OD}_{600}=1.0$ were injected by the syringe-infiltration method into 4-leaf stage plants of different cultivars, A5 (CM 334), A3 (PBC 137), and EC (Early Calwonder). Then, inoculated pepper plants were transferred to $18^{\circ} \mathrm{C}$ for 2 days and grown in a growth chamber at $22^{\circ} \mathrm{C}$ under a $16-\mathrm{h}$ light $/ 8$-h dark photoperiod cycle with $60 \%$ relative humidity. The efficiency of silencing $C a P D S$ was analyzed at 15 days postinoculation, which was calculated in accordance with the formula described in Statistical analysis. Letters indicate significant differences using the Fisher LSD test at $\mathrm{P} \leq 0.05$.

\section{Effect of plant growth stage on VIGS efficiency in agroinoculated pepper}

The developmental stage of a plant at the time of inoculation is a known critical factor for VIGS (Chung et al., 2004; Hileman et al., 2005; Burch-Smith et al., 2006; Deng et al., 2012). To investigate the effect of the growth stage of agroinoculated pepper plants on gene silencing efficiency, we attempted to silence the gene at different growth stages (2-, 4-, 6-, and 8-leaf) in the EC cultivar. The Agrobacterium inoculum, with a final $\mathrm{OD}_{600}$ of 1.0 , was injected into pepper leaves through the syringe-infiltration method. Plants were then grown at $22^{\circ} \mathrm{C}$ under a 16 -h light $/ 8$-h dark photoperiod cycle with $60 \%$ relative humidity. The CaPDS gene silencing efficiency results are shown in Figure 2. The photobleaching symptom was observed on the leaves of 2-, 4-, and 6-leaf stage plants at 15 days postinoculation, and gene silencing efficiencies were 96,90 , and $58 \%$, respectively. In contrast, leaves of 8 -leaf stage plants showed no obvious photobleaching at this time, which was observed 10 days later than the phenotype first appeared in 2-leaf stage plants. These results demonstrate that younger plants are preferable for TRV-based silencing in pepper. Therefore, because the 2-leaf stage of pepper plants was the most competent for Agrobacterium infection, it was used in subsequent experiments for gene silencing. 


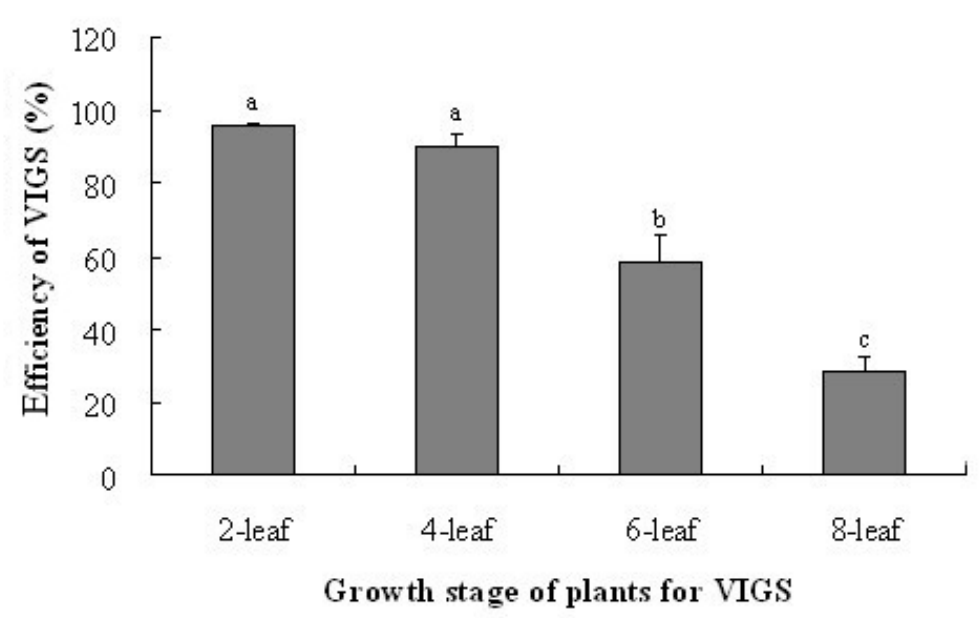

Figure 2. Effect of different growth stage of plants for agroinoculation on virus-induced gene silencing (VIGS) efficiency in pepper. The agroinocula with a final $\mathrm{OD}_{600}=1.0$ were injected into different growth (2-, 4-, 6-, 8-leaf) stage plants through the syringe-infiltration method, which were then grown at $22^{\circ} \mathrm{C}$ under a 16 -h light/ 8 -h dark photoperiod cycle with $60 \%$ humidity. The efficiency of silencing $C a P D S$ was analyzed at 15 days postinoculation, which was calculated in accordance with the formula described in Statistical analysis. Letters indicate significant differences using the Fisher LSD test at $\mathrm{P} \leq 0.05$.

\section{Effect of different concentrations of agroinocula on VIGS efficiency in pepper}

Previous studies have shown that in some plant species, the concentration of agroinoculation affects gene silencing efficiency (Chung et al., 2004; Burch-Smith et al., 2006; Zhou et al., 2006). In order to determine the optimal agroinoculation concentration, different concentrations of Agrobacterium inocula ( $\mathrm{OD}_{600}$ values of $0.5,0.8,1.0,2.0$, and 3.0) were syringeinfiltrated into 2-leaf stage EC plants, which were then grown in growth chambers at $22^{\circ} \mathrm{C}$. The resulting $C a P D S$ gene silencing efficiencies from the different concentrations of agroinocula are shown in Figure 3. The highest gene silencing efficiencies were 88 and $83 \%$, with final $\mathrm{OD}_{600}$ of 1.0 and 0.8 , respectively. When the agroinoculation concentration was lower than an $\mathrm{OD}_{600}$ of 0.8 , the gene silencing efficiency decreased to $47 \%$. When the concentration of agroinoculation was $\mathrm{OD}_{600}=2.0$, the gene silencing efficiency was $38 \%$. In addition, higher death rates occurred on plants infected with a final $\mathrm{OD}_{600}$ of 3.0 . These results confirmed that a concentration with a final $\mathrm{OD}_{600}$ of 1.0 was suitable for gene silencing in pepper plants.

\section{Effect of different growth temperatures of agroinoculated plants on VIGS efficiency in pepper}

The growth temperature of agroinoculated plants is another important factor that influences several aspects in VIGS, such as Agrobacterium performance, viral spread, accumulation of shortinterfering RNAs, and ultimately, plant growth (Burch-Smith et al., 2004; Robertson, 2004; Hartl et al., 2008). The particular effect of temperature is species-specific. For example, several Solanum spp favor temperatures between $16^{\circ}$ and $20^{\circ} \mathrm{C}$ (Liu et al., 2002a; Brigneti et al., 2004), whereas $A$. thaliana favors higher temperatures, between $22^{\circ}$ and $24^{\circ} \mathrm{C}$ (Wang et al., 2006). 
To assess the influence of the growth temperature of agroinoculated pepper plants on gene silencing, 2-leaf stage agroinoculated plants, with a final $\mathrm{OD}_{600}$ of 1.0 , were grown in plant growth chambers at different temperatures $\left(18^{\circ}, 20^{\circ}, 22^{\circ}, 25^{\circ}\right.$, and $\left.28^{\circ} \mathrm{C}\right)$. The results of temperature on the efficiency of $C a P D S$ gene silencing are shown in Figure 4. The pTRV2-PDS-silenced plants grown at $20^{\circ}, 22^{\circ}$, and $25^{\circ} \mathrm{C}$ demonstrated the highest gene silencing efficiencies of 86,88 , and $80 \%$, respectively, while plants grown at the lowest $\left(18^{\circ} \mathrm{C}\right)$ and the highest $\left(28^{\circ} \mathrm{C}\right)$ temperatures displayed the lowest gene silencing efficiencies; 28 and $11 \%$, respectively.

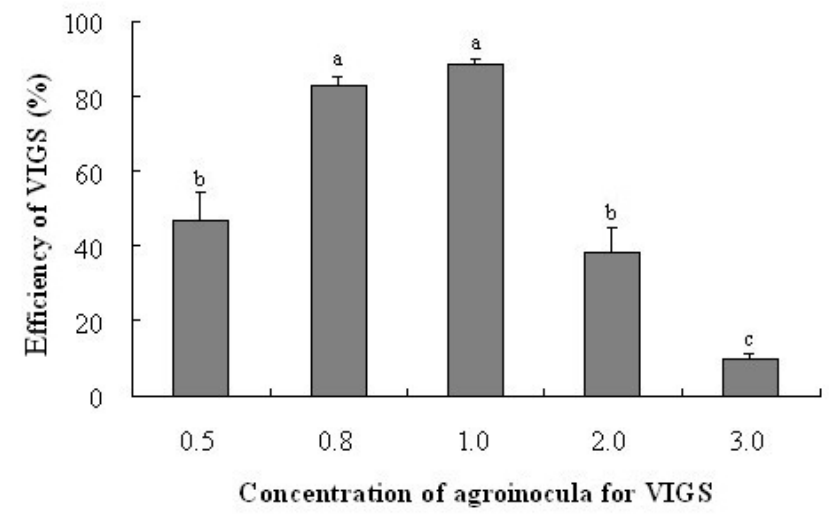

Figure 3. Effect of the different concentrations of agroinocula for agroinoculation on virus-induced gene silencing (VIGS) efficiency in pepper. The agrocultures with different concentration $\left(\mathrm{OD}_{600}=0.5,0.8,1.0,2.0,3.0\right)$ were injected into 2-leaf stage plants through the syringe-infiltration method, which were then grown at $22^{\circ} \mathrm{C}$ under a 16-h light/8-h dark photoperiod cycle with $60 \%$ humidity. The efficiency of silencing $C a P D S$ was analyzed at 15 days postinoculation, which was calculated in accordance with the formula described in Statistical analysis. Letters indicate significant differences using the Fisher LSD test at $\mathrm{P} \leq 0.05$.

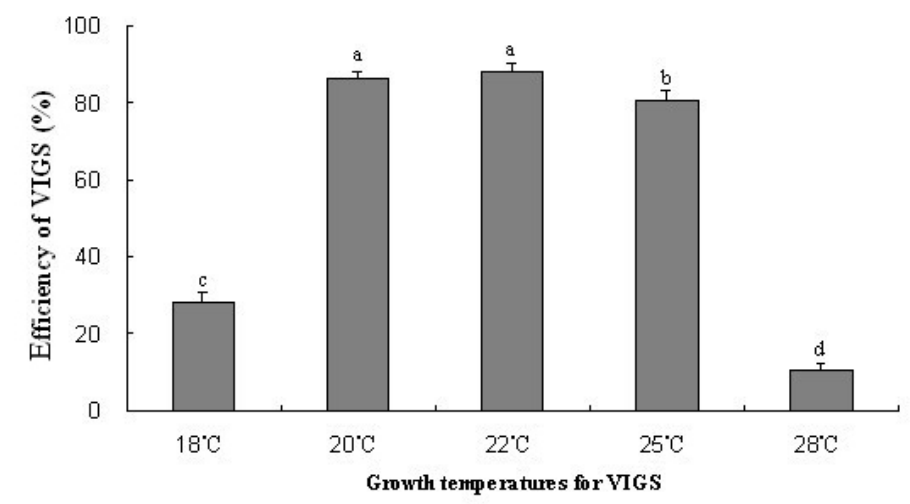

Figure 4. Effect of the different growth temperatures of agroinoculated plants on virus-induced gene silencing (VIGS) efficiency in pepper. The agrocultures with a final $\mathrm{OD}_{600}=1.0$ were injected into 2-leaf stage plants through the syringe-infiltration method, which were then grown at different temperatures $\left(18^{\circ}, 20^{\circ}, 22^{\circ}, 25^{\circ}\right.$, and $\left.28^{\circ} \mathrm{C}\right)$ under a 16-h light/8-h dark photoperiod cycle with $60 \%$ humidity. The efficiency of silencing $C a P D S$ was analyzed at 15 days postinoculation, which was calculated in accordance with the formula described in Statistical analysis. Letters indicate significant differences using the Fisher LSD test at $\mathrm{P} \leq 0.05$. 
In addition, the earliest sign of photobleaching was observed in agroinoculated plants grown at $22^{\circ} \mathrm{C}$, whereas the silencing phenotype only appeared on plants grown at $20^{\circ}$ and $25^{\circ} \mathrm{C} 2-3$ days later. The expression of the photobleaching symptom was delayed for 7-10 days in plants grown at $18^{\circ}$ and $28^{\circ} \mathrm{C}$, which had substantially reduced leaf areas relative to those grown at $22^{\circ} \mathrm{C}$. These results demonstrated that $22^{\circ} \mathrm{C}$ was the optimal temperature for plant growth after agroinoculation, which is within the natural temperature range of pepper growth.

\section{VIGS of CaPDS in pepper}

To assess the general effectiveness of the TRV-based VIGS protocol for gene silencing that was optimized in the pepper cultivar EC, we examined $C a P D S$ gene silencing in 3 other pepper cultivars: A5, A3, and B12. The optimized protocol for gene silencing in pepper involves agroinoculating, via syringe-infiltration, Agrobacterium with an $\mathrm{OD}_{600}$ of 1.0 into 2-leaf growth stage plants at $22^{\circ} \mathrm{C}$. Following this protocol, the photobleaching symptom was exhibited in all inoculated pepper plants just as was observed in EC (Figure 5A). These results therefore demonstrate that the optimized protocol for gene silencing in the EC cultivar is suitable for other cultivars of pepper.

Furthermore, we used chlorophyll content as another measure of silencing efficiency. More chlorophyll degradation indicates higher silencing efficiency and vice versa. Chlorophyll content was assessed in the third or fourth leaf from the upper infected leaves showing the photobleaching phenotype, and was compared with the chlorophyll content of leaves from the mock-inoculated plants (pTRV-00; empty vector) that did not show photobleaching. As shown in Figure 5B, the contents of chlorophyll a, chlorophyll b, and total chlorophyll in the leaves of $C a P D S$-silenced plants were substantially reduced relative to those of control plants (pTRV-00; empty vector). The highest reductions in total chlorophyll were observed in the CaPDS-silenced leaves of EC (86\%) and B12 (54\%), whereas the silenced leaves of A5 and A3 showed only 38 and $44 \%$ reductions, respectively.

To further confirm gene silencing of CaPDS at the molecular level, qRT-PCR was performed with gene specific primers (see Materials and Methods for details). Total RNA was extracted from the third or fourth photobleached leaf from the upper leaves of inoculated plants and from the non-photobleached leaves of the control plants (pTRV-00; empty vector). The results of gene expression patterns are shown in Figure 5C. The expression of the CaPDS genes in silenced plants A5, A3, EC, and B12 were dramatically reduced 51, 75, 91, and 85\%, respectively, compared to control plants. These results further demonstrate that the optimized protocol efficiently silences $C a P D S$ in pepper plants.

\section{Virus-induced gene silencing of $\mathrm{CaPOD}$ in pepper}

To validate that the gene silencing protocol optimized for $C a P D S$ is also applicable to other genes, we attempted to silence the $C a P O D$ gene in the EC cultivar. The optimized protocol for gene silencing in pepper involves agroinoculating, via syringe-infiltration, Agrobacterium with an $\mathrm{OD}_{600}$ of 1.0 into 2-leaf growth stage plants at $22^{\circ} \mathrm{C}$. Following this protocol, the CaPOD-inoculated EC plants exhibited viral symptoms (Figure 6A). This result demonstrated that the optimized protocol in EC is also applicable to other genes for virus-induced gene silencing in pepper plants. 


\section{A}
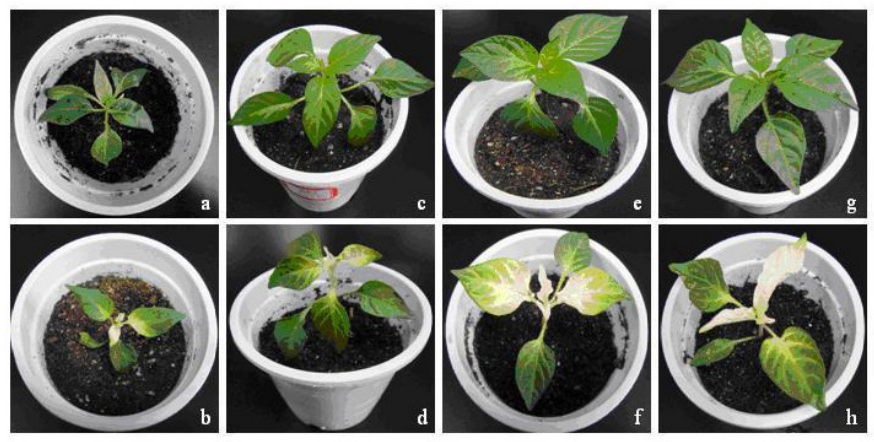

B
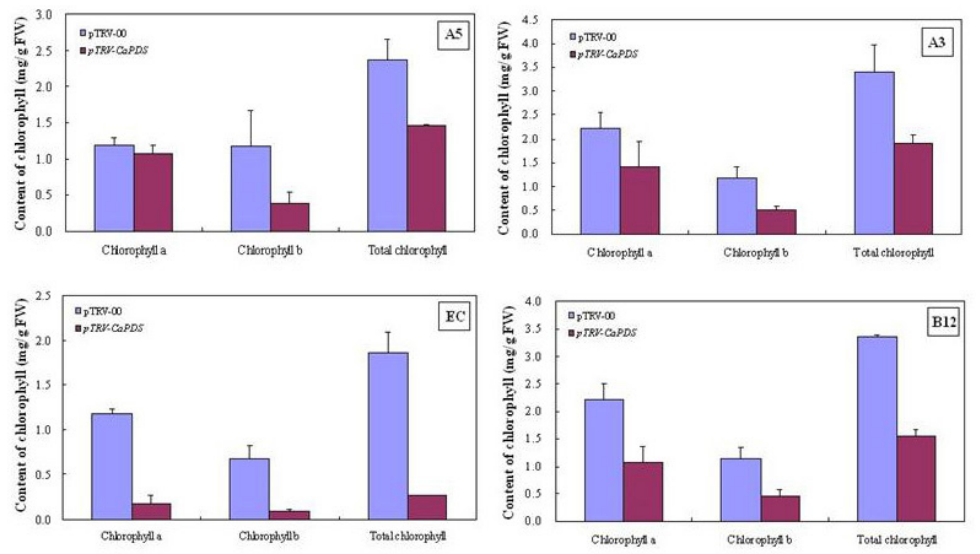

C
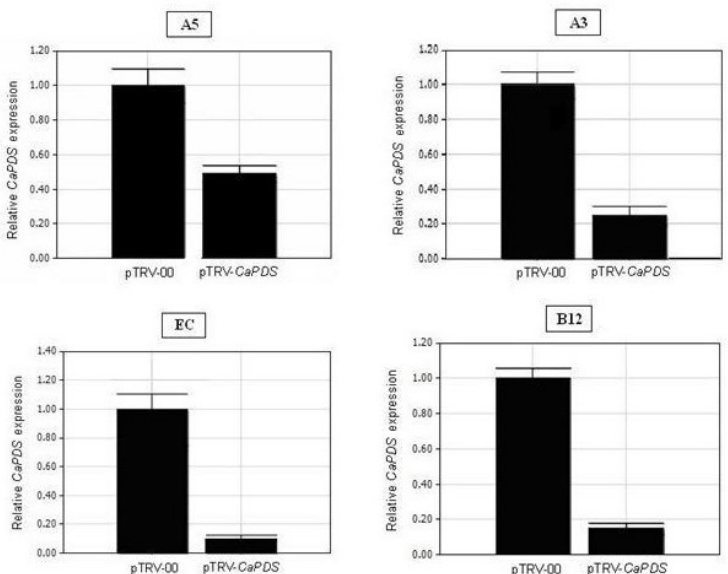

Figure 5. TRV-induced gene silencing of the CaPDS gene in 4 cultivars of pepper. The photographs were taken 20 days postinoculation. A. Symptoms of inoculated plants: a. control plant (pTRV-00, empty vector) of A5; b. pTRV-CaPDS-infected plant of A5; c. control plant (pTRV-00, empty vector) of A3; d. pTRV-CaPDS-infected plant of A3; e. control plant (pTRV-00, empty vector) of EC; f. pTRV-CaPDS-infected plant of EC; g. control plant (pTRV-00, empty vector) of B12; h. pTRV-CaPDS-infected plant of B12. B. Comparison of the content of chlorophyll: chlorophyll $\mathrm{a}$; chlorophyll $\mathrm{b}$, and total chlorophyll between the control (pTRV-00, empty vector) and pTRV-CaPDS-silenced plants. C. Real-time RT-PCR analysis of CaPDS gene expression in the control (pTRV-00, empty vector) and pTRV-CaPDS-silenced plants. 
To confirm the silencing of the $C a P O D$ gene at the molecular level, expression of the CaPOD gene was determined by RT-PCR. Total RNA was extracted from the third or fourth leaves of injected and control (pTRV-00; empty vector) plants. Expression of CaPOD genes in infected plants decreased by more than $26 \%$ compared to that of control plants (Figure 6B). These results further demonstrate that the optimized protocol is efficient for silencing other genes in pepper plants.
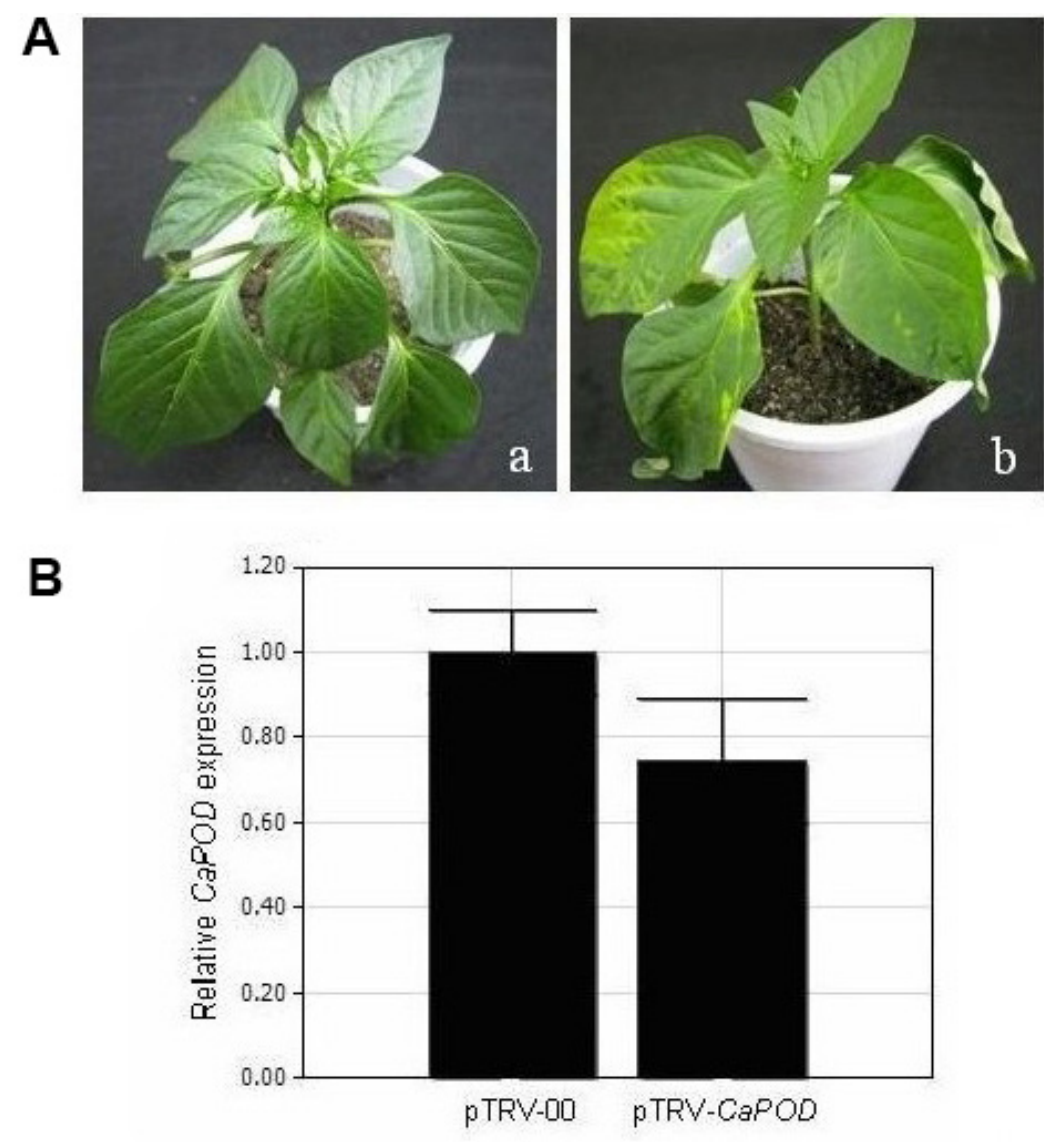

Figure 6 TRV-induced gene silencing of $C a P O D$ in pepper cultivar Early Calwonder. A. Symptoms of inoculated plants. The photographs were taken 30 days postinoculation. a. control plant (pTRV-00, empty vector); b. pTRV$\mathrm{CaPOD}$-infected plant. B. Real-time RT-PCR analysis of $\mathrm{CaPOD}$ gene expression in the control (pTRV-00, empty vector) and pTRV-CaPOD-silenced plants.

\section{DISCUSSION}

VIGS technology has shown its potential as a reverse genetic tool, especially for plants lacking a high throughput transformation system. This is mainly because VIGS is a very simple and robust method that does not require stable transformation and extensive gene 
sequence information (Sasaki et al., 2011). Successful gene silencing depends upon a dynamic interplay between virus spread and plant growth, both of which can be influenced by environmental conditions. Factors related to the rapid accumulation of the TRV can greatly affect the efficiency of TRV-induced gene silencing. In particular, most authors have emphasized the choice of vector, the inoculation method, plant age, and plant growth temperature as key parameters (Brigneti et al., 2004; Saedler and Baldwin, 2004; Burch-Smith et al., 2006; Wang et al., 2006). It is, therefore, important to invest a substantial amount of effort to determine optimal inoculation levels and plant growth conditions prior to conducting VIGS studies (BurchSmith et al., 2004). However, once these factors are optimized, it is fairly simple to obtain reproducible levels of silencing plants.

In this study, we optimized a TRV-based virus-induced gene silencing protocol via syringe-infiltration for pepper plants. The factors affecting the efficiency of VIGS in pepper plants included the specific cultivar used, growth stage, the concentration of Agrobacterium inoculum, and the growth temperature. Several studies have shown that TRV infection causes relatively mild disease symptoms and often induces intensive and uniform silencing phenotypes (Ratcliff et al., 2001; Brigneti et al., 2004; Burch-Smith et al., 2006). Therefore, the TRV was used for silencing pepper plant genes in our experiment.

Genetic differences among cultivars of the same species may result in differences in their susceptibility to TRV infection (Ghazala and Varrelmann, 2007; MacFarlane, 2010) or TRV-based virus-induced gene silencing (Chen et al., 2004). We confirmed this observation as the different pepper cultivars sampled showed large variations in their TRV-based gene silencing responses. EC showed obvious photobleaching symptoms 13 days postinoculation, whereas A5 exhibited this silencing phenotype 7 days later.

The developmental stage of the plant at the time of inoculation was also found to strongly affect silencing efficiency (Burch-Smith et al., 2006; Deng et al., 2012). VIGS has mostly been used to investigate gene function in young seedlings or during seedling development (Liu et al., 2002; Chung et al., 2004). This implies that the host plant may have a particularly competent stage for Agrobacterium infiltration. Through comparisons of inoculation via syringe-infiltration across life stages, we concluded that young seedlings of pepper plants show the highest efficiency in gene silencing. The reason may be that the Agrobacterium inoculum is more easily injected in younger leaves.

It has previously been shown that the concentration of agroinoculation affects gene silencing efficiency in some plant species (Chung et al., 2004; Burch-Smith et al., 2006; Zhou et al., 2006). From our results, the Agrobacterium inoculum with a final $\mathrm{OD}_{600}$ of 1.0 was the most efficient for gene silencing in pepper plants. When the Agrobacterium concentration was lower than 1.0, the efficiency of plant infection was low and the photobleaching symptom was not evident. In contrast, when the Agrobacterium concentration was higher than 2.0, the host plants expressed more dead blots on the leaves. This is probably because, under these optical conditions, the infectious TRV is more rapidly available in plant cells, causing increased infection.

Temperature is one of the most important factors for good viral spread and effective silencing (Burch-Smith et al., 2004). Several Solanum species favor lower temperatures, ranging from $16^{\circ}$ to $20^{\circ} \mathrm{C}$ (Liu et al., 2002a; Brigneti et al., 2004), but the best results in A. thaliana are achieved at temperatures of $23^{\circ} \mathrm{C}$ or higher. Therefore, temperature is an important consideration when optimizing VIGS in new plants species. In our experiment, we observed that the development of good silencing phenotypes with TRV on pepper occurs at $22^{\circ} \mathrm{C}$, and it 
must be grown at $18^{\circ} \mathrm{C}$ for 2 days after injection. The low temperature treatment after injection may actually be one of the contributing reasons for the high efficiency observed (Chung et al., 2004). In fact, plants generally grew more vigorously under cooler conditions. Additionally, Fu et al. (2005) showed that conditions of low temperature $\left(15^{\circ}-18^{\circ} \mathrm{C}\right)$ and low humidity $(30-$ $40 \%$ ) enhanced the silencing of $P D S$ throughout inoculated tomato plants, flowers, and fruits.

The silencing phenotype of $P D S$ has been used as a visual marker for the effectiveness of VIGS in several plants, such as tomato (Liu et al., 2002a), A. thaliana (Turnage et al., 2002), $N$. benthamiana (Ratcliff et al., 2001), and pepper (Chung et al., 2004). The silencing of PDS produces a typical white color, which occurs in the absence of the PDS gene product. Using this optimal protocol, we achieved highly efficient VIGS for CaPDS in 4 pepper cultivars (Figure 5A). Moreover, to further verify the efficiency of VIGS, we also examined the chlorophyll content of inoculated plants (Figure 5B). More chlorophyll degradation indicates higher silencing efficiency, and vice versa. Finally, RT-PCR was performed (Figure 5C). From these results, we concluded that the optimized protocol is applicable to other cultivars of pepper. Furthermore, silencing of another gene, $C a P O D$, which is involved in the defense response to pathogens, was also investigated (Figure 6). At 30 days postinoculation, the pTRV-CaPODinoculated plants showed viral symptoms, but the control plants (pTRV-00; empty vector) had not changed. Therefore, VIGS can likely be used to exploit several significant genes in pepper, such as genes related to disease-resistance.

\section{CONCLUSIONS}

VIGS is a widely used, powerful technique for reverse genetics. Vectors derived from the TRV are among the most popular for VIGS. In this study, we optimized the protocol for TRV-induced gene silencing in pepper plants. The optimized TRV-based protocol can be used to effectively silence the reporter gene, CaPDS, in different pepper cultivars. Importantly, TRV-based VIGS is also efficient in silencing $C a P O D$, which is a gene involved in the defense response to pathogens. Therefore, this TRV-based gene silencing system provides a rapid and high-throughput technique platform for gene function analysis in pepper.

\section{ACKNOWLEDGMENTS}

Research supported by the National Natural Science Foundation of China (\#31272163), "The Twelfth Five-Year" Plan of National Science and Technology in Rural Areas (\#2011BAD12B03), and the Shaanxi Provincial Science and Technology Coordinating Innovative Engineering Project (\#2012KTCL02-09).

\section{REFERENCES}

\footnotetext{
Baulcombe D (1999). Viruses and gene silencing in plants. Arch. Virol. Suppl. 15: 189-201. Baulcombe D (2004). RNA silencing in plants. Nature 431: 356-363.

Becker A and Lange M (2010). VIGS - genomics goes functional. Trends Plant Sci. 15: 1-4.

Benedito VA, Visser PB, Angenent GC and Krens FA (2004). The potential of virus-induced gene silencing for speeding up functional characterization of plant genes. Genet. Mol. Res. 3: 323-341.

Bennypaul HS, Mutti JS, Rustgi S, Kumar N, et al. (2012). Virus-induced gene silencing (VIGS) of genes expressed in root, leaf, and meiotic tissues of wheat. Funct. Integr. Genomics 12: 143-156.
} 
Brigneti G, Martin-Hernandez AM, Jin H, Chen J, et al. (2004). Virus-induced gene silencing in Solanum species. Plant J. 39: 264-272.

Burch-Smith TM, Anderson JC, Martin GB and Dinesh-Kumar SP (2004). Applications and advantages of virus-induced gene silencing for gene function studies in plants. Plant J. 39: 734-746.

Burch-Smith TM, Schiff M, Liu Y and Dinesh-Kumar SP (2006). Efficient virus-induced gene silencing in Arabidopsis. Plant Physiol. 142: 21-27.

Chen JC, Jiang CZ, Gookin TE, Hunter DA, et al. (2004). Chalcone synthase as a reporter in virus-induced gene silencing studies of flower senescence. Plant Mol. Biol. 55: 521-530.

Chung E, Seong E, Kim YC, Chung EJ, et al. (2004). A method of high frequency virus-induced gene silencing in chili pepper (Capsicum annuum L. cv. Bukang). Mol. Cells 17: 377-380.

Cunningham FX and Gantt E (1998). Genes and enzymes of carotenoid biosynthesis in plants. Annu. Rev. Plant Physiol. Plant Mol. Biol. 49: 557-583.

Deng X, Elomaa P, Nguyen CX, Hytonen T, et al. (2012). Virus-induced gene silencing for Asteraceae--a reverse genetics approach for functional genomics in Gerbera hybrida. Plant Biotechnol. J. 10: 970-978.

Dinesh-Kumar SP, Anandalakshmi R, Marathe R, Schiff M, et al. (2003). Virus-induced gene silencing. Methods Mol. Biol. 236: 287-294.

Ding XS, Schneider WL, Chaluvadi SR, Mian MA, et al. (2006). Characterization of a Brome mosaic virus strain and its use as a vector for gene silencing in monocotyledonous hosts. Mol. Plant Microbe Interact. 19: 1229-1239.

Fu DQ, Zhu BZ, Zhu HL, Jiang WB, et al. (2005). Virus-induced gene silencing in tomato fruit. Plant J. 43: 299-308.

Ghazala W and Varrelmann M (2007). Tobacco rattle virus $29 \mathrm{~K}$ movement protein is the elicitor of extreme and hypersensitive-like resistance in two cultivars of Solanum tuberosum. Mol. Plant Microbe Interact. 20: 1396-1405.

Hajirezaei MR, Peisker M, Tschiersch H, Palatnik JF, et al. (2002). Small changes in the activity of chloroplastic NADP ${ }_{-}$ dependent ferredoxin oxidoreductase lead to impaired plant growth and restrict photosynthetic activity of transgenic tobacco plants. Plant J. 29: 281-293.

Hartl M, Merker H, Schmidt DD and Baldwin IT (2008). Optimized virus-induced gene silencing in Solanum nigrum reveals the defensive function of leucine aminopeptidase against herbivores and the shortcomings of empty vector controls. New Phytol. 179: 356-365.

Hileman LC, Drea S, Martino G, Litt A, et al. (2005). Virus-induced gene silencing is an effective tool for assaying gene function in the basal eudicot species Papaver somniferum (opium poppy). Plant J. 44: 334-341.

Holzberg S, Brosio P, Gross C and Pogue GP (2002). Barley stripe mosaic virus-induced gene silencing in a monocot plant. Plant J. 30: 315-327.

Huang C, Qian Y, Li Z and Zhou X (2012). Virus-induced gene silencing and its application in plant functional genomics. Sci. China Life Sci. 55: 99-108.

Kim S, Kim SR, An CS, Hong YN, et al. (2001). Constitutive expression of rice MADS box gene using seed explants in hot pepper (Capsicum annuum L.). Mol. Cells 12: 221-226.

Kumagai MH, Donson J, della-Cioppa G, Harvey D, et al. (1995). Cytoplasmic inhibition of carotenoid biosynthesis with virus-derived RNA. Proc. Natl. Acad. Sci. U. S. A. 92: 1679-1683.

Lichtenthaler HK (1987). Chlorophylls and carotenoids: pigments of photosynthetic biomembranes. Meth. Enzymol. 148: 350-382.

Liu H, Fu D, Zhu B, Yan H, et al. (2012). Virus-induced gene silencing in eggplant (Solanum melongena). J. Integr. Plant Biol. 54: 422-429.

Liu Y, Schiff M and Dinesh-Kumar SP (2002a). Virus-induced gene silencing in tomato. Plant J. 31: 777-786.

Liu Y, Schiff M, Marathe R and Dinesh-Kumar SP (2002b). Tobacco Rar1, EDS1 and NPR1/NIM1 like genes are required for N-mediated resistance to tobacco mosaic virus. Plant J. 30: 415-429.

Liu Y, Nakayama N, Schiff M, Litt A, et al. (2004). Virus induced gene silencing of a DEFICIENS ortholog in Nicotiana benthamiana. Plant Mol. Biol. 54: 701-711.

MacFarlane SA (2010). Tobraviruses - plant pathogens and tools for biotechnology. Mol. Plant Pathol. 11: 577-583.

Manoharan M, Vidya CS and Sita GL (1998). Agrobacterium-mediated genetic transformation in hot chilli (Capsicum annuum L. var. Pusa jwala). Plant Sci. 131: 77-83.

Mihalka V, Fari M, Szasz A, Balazs E, et al. (2000). Optimized protocols for efficient regeneration and gene transfer in pepper (Capsicum annuum L.). J. Plant Biotechnol. 2: 143-149.

Orzaez D, Mirabel S, Wieland WH and Granell A (2006). Agroinjection of tomato fruits. A tool for rapid functional analysis of transgenes directly in fruit. Plant Physiol. 140: 3-11.

Ratcliff F, Martin-Hernandez AM and Baulcombe DC (2001). Technical Advance. Tobacco rattle virus as a vector for analysis of gene function by silencing. Plant J. 25: 237-245.

Robertson D (2004). VIGS vectors for gene silencing: many targets, many tools. Annu. Rev. Plant Biol. 55: 495-519. 
Ruiz MT, Voinnet O and Baulcombe DC (1998). Initiation and maintenance of virus-induced gene silencing. Plant Cell 10: 937-946.

Saedler R and Baldwin IT (2004). Virus-induced gene silencing of jasmonate-induced direct defences, nicotine and trypsin proteinase-inhibitors in Nicotiana attenuata. J. Exp. Bot. 55: 151-157.

Sasaki S, Yamagishi N and Yoshikawa N (2011). Efficient virus-induced gene silencing in apple, pear and Japanese pear using Apple latent spherical virus vectors. Plant Methods 7: 15.

Turnage MA, Muangsan N, Peele CG and Robertson D (2002). Geminivirus-based vectors for gene silencing in Arabidopsis. Plant J. 30: 107-114.

Valentine T, Shaw J, Blok VC, Phillips MS, et al. (2004). Efficient virus-induced gene silencing in roots using a modified tobacco rattle virus vector. Plant Physiol. 136: 3999-4009.

Wang CC, Cai XZ, Wang XM and heng Z (2006). Optimisation of tobacco rattle virus-induced gene silencing in Arabidopsis. Functional Plant Biol. 33: 347-355.

Zhou XF, Ma PD, Wang RH, Liu B, et al. (2006). A novel approach to functional analysis of the ribulose bisphosphate carboxylase small subunit gene by Agrobacterium-mediated gene silencing. J. Integrative Plant Biol. 48: 1225-1232. 